\title{
Germanica
}

\section{Eine Kiste, deren Inhalt nicht mehr zugänglich ist - Wolfgang Hilbigs Roman Das Provisorium und sein Umgang mit der Shoah}

Une caisse dont le contenu n'est plus accessible - le roman de Wolfgang Hilbig

Das Provisorium et son rapport à la Shoah

A box whose content is no longer accessible - Wolfgang Hilbig's novel Das

Provisorium and its treatment of the Shoah

\section{Florian Gödel}

\section{OpenEdition}

\section{Journals}

Édition électronique

URL : http://journals.openedition.org/germanica/3440

DOI : $10.4000 /$ germanica. 3440

ISSN : 2107-0784

Éditeur

Université de Lille

Édition imprimée

Date de publication : 30 décembre 2016

Pagination : 99-114

ISBN : 9782913857384

ISSN : 0984-2632

\section{Référence électronique}

Florian Gödel, „Eine Kiste, deren Inhalt nicht mehr zugänglich ist - Wolfgang Hilbigs Roman Das

Provisorium und sein Umgang mit der Shoah", Germanica [Online], 59 | 2016, Online erschienen am: 30 Dezember 2018, abgerufen am 06 Januar 2021. URL: http://journals.openedition.org/germanica/3440 ; DOI: https://doi.org/10.4000/germanica.3440 


\title{
Eine Kiste, deren Inhalt nicht mehr zugänglich ist - Wolfgang Hilbigs Roman Das Provisorium und sein Umgang mit der Shoah
}

\author{
Florian Gödel \\ Friedrich-Schiller-Universität Jena
}

Wolfgang Hilbig hat sich stets als ein Autor hervorgetan, der durch seine Nonkonformität in der DDR zwangsläufig politisch werden musste. Andererseits versteht er selbst sein Schreiben als einen beinahe biologischen Zwang, so etwa wenn er von einer existenzialistischen Unruhe spricht, die den Dichter im Allgemeinen und auch den Autor Hilbig ausmacht ${ }^{1}$. Das lässt vermuten, dass sich in seinem Werk politisch-historische Problematiken mit einer Identitäts- und Individualitätsproblematik überkreuzen. Die Frage nach der eigenen Identität scheint - zumindest in diesem Falle - eng an die Frage nach dem Schreiben geknüpft zu sein, wobei die Krise des Individuums einerseits Bedingung für das Schreiben ist und andererseits die Grenzen des Schreibbaren eng umreißt. Eine besondere Bedeutung erlangt die Überkreuzung dieser Problemstellungen in Hilbigs letztem großen Roman Das Provisorium. Das liegt daran, dass hier der Schreibende selbst im Mittelpunkt steht. Gewissermaßen ist Das Provisorium noch

1. - Vgl. Sylvie Marie Bordaux, Literatur als Subversion. Eine Untersuchung des Prosawerkes von Wolfgang Hilbig, Göttingen, Cuvillier, 2000, S. 5. 
einmal ein Musterbeispiel für einen selbstreflexiven Roman. Auch wenn der Autor die erste Person Singular der ersten handschriftlichen Version später in die dritte Person des Schriftstellers C. abändert, bleibt seine Position zum Text die ursprüngliche: „Es war mir auch im ,Provisorium" immer klar, dass ich mich beschreibe“2. Das bringt Schwierigkeiten in Bezug auf die Analyse des Textes mit sich, die trotz der engen Verbindungen, welche der Autor selbst betont, die grundlegende Trennung zwischen Protagonist und Autor aufrechterhalten muss.

Die These dieses Artikels ist also, dass sich die Überkreuzung beider Problemstellungen in einem Punkt beobachten lässt. Dieser Punkt soll hier der darstellerische Umgang mit der Shoah sein. Die Art, wie der Protagonist mit ihr in Berührung kommt und wieder von ihr abdriftet als Politikum, scheinbar gesellschaftliche Psychose, als innere Stimme und Kategorie fragwürdiger Relativierung - führt zurück auf die Kernfrage, die mit Adornos Satzfragment ,nach Auschwitz ein Gedicht zu schreiben, ist barbarisch [... $]^{3 \text { " }}$ virulent wurde: Wie und insbesondere mit welchen literarischen Methoden ist Schreiben nach Auschwitz möglich? Was bedeutet diese poetologische Normativität ganz neuen Ausmaßes für das schreibende Individuum?

Der Roman gestaltet sich überwiegend als ein Rückblick auf das ewig provisorische Leben des Schriftstellers C. zwischen den Systemen des geteilten Deutschland und der Nachwendezeit. Die Shoah taucht hier, meist im Verbund mit dem Gulag, an zentralen Textstellen auf, wenn es um das verlogene Jahrhundert geht, das in der Konsequenz aus C. selbst einen Lügner machte, um noch Schriftsteller sein zu können. Hier wird vor allem der poetologische Aspekt im Umgang mit der Shoah interessant: Mit Adorno ist Auschwitz zur Begründung für die Schreibweisen der Moderne ${ }^{4}$ geworden und beerbt damit den Begriff der Revolution, sei es die poetische Revolution bei Arthur Rimbaud oder die revolutionäre Geburt des Dada aus dem Ersten Weltkrieg. Auf Auschwitz folgte keine literarische Bewegung, die dem Dada und Surrealismus vergleichbar wäre. Trotzdem wird die Literatur immer wieder auf Auschwitz zurück-

2. - Jürgen Hosemann, „Nachbemerkung zu dieser Ausgabe“, in: Wolfgang Hilbig, Das Provisorium. Roman. Mit einem Nachwort von Julia Frank, Frankfurt a.M., S. Fischer, 2013, S. 321 (Hervorhebung im Original).

3. - Zitiert nach Peter Stein, ,'Darum mag falsch gewesen sein, nach Auschwitz ließe kein Gedicht sich schreiben." (Adorno). Widerruf eines Verdikts? Ein Zitat und seine Verkürzung“, in: Weimarer Beiträge, 1996, H. 4, S. 485. Stein geht ausführlich auf die problematische Rezeptionsgeschichte des Adorno-Zitates ein und verweist auch auf die verfälschte Übernahme des Diktums in der DDR der 60er Jahre.

4. - Vor allem scheint dies zu gelten für eine Hinwendung ,moderner Literatur“ zu Fragen nach dem Individuum. Insofern ist für Adorno Auschwitz eine Neubegründung der literarischen Moderne, weil die gesellschaftliche Kollektivierung als zentrale Bedingung für Auschwitz erkannt worden ist. Vgl. Theodor W. Adorno, Kulturkritik und Gesellschaft, Frankfurt a.M., Suhrkamp, 1977, S. 681 f. 
verwiesen, wenn sie sich die Frage nach der Abbildbarkeit der Realität in einer selbstreflexiven und autoreferenziellen Sprache stellt. Hayden White schließlich lässt nur moderne Darstellungsweisen als angemessen für die Behandlung der Shoah gelten.

[T]he kinds of anti-narrative non-stories produced by literary modernism offer the only prospect for adequate representations of the kind of ,unnatural“" events - including the Holocaust - that mark our era and distinguish it absolutely from all the "history“ that has come before $\mathrm{it}^{5}$.

Im Provisorium treten Shoah und Gulag ganz plastisch hervor nicht nur im Sinne der zwei Bücherkisten, die der Schriftsteller C. mit der Etikette „Holocaust \& Gulag“ versehen hat ${ }^{6}$ - vielmehr widmet sich Hilbig ganz offen der Bedeutung von Auschwitz für seine schriftstellerische Existenz. C.s Kampf gegen den Alkohol, gegen die schriftstellerische und sexuelle Impotenz, gegen Gott und Staatsapparate gerät zum fieberhaften Versuch, die Ursprünge des Schreibens in der Kindheit aufzudecken und sich damit der idealischen Berufung zum Dichtertum zu vergewissern. Der zentrale Begriff für C.s Dasein ist die Lüge, die er anderen auflastet, um sich der größeren Lüge, den zwei mörderischen Ideologien des 20. Jahrhunderts, zu entziehen $(D P, 255)$. Die Auseinandersetzung mit der schriftstellerischen Legitimation läuft für C. auf die Konfrontation mit den fundamentalen Verbrechen des Jahrhunderts und der großen Lüge als ihres Ermöglichungsgrundes hinaus.

$\mathrm{Zu}$ Beginn soll nach intertextuellen Verweisen auf die literarische Moderne innerhalb des Romans gesucht werden, um die Vorbildrolle moderner Autoren aufzudecken. Anschließend wird der Bogen zur Darstellung der Shoah geschlagen. Es wird gezeigt, wie diese mit dem Selbstverständnis des schreibenden Individuums und dem Konzept der Lüge zusammenhängt. Es folgt eine Untersuchung der ShoahDarstellung unter dem Aspekt ihrer Relativierung durch den Gulag einerseits und die beiden deutschen Staaten andererseits. Schließlich wird im Anschluss daran kurz auf die Frage eingegangen, ob die Shoah noch immer einen Status von Einzigartigkeit innehat, um somit die Beobachtungen zusammenzuführen. Dabei muss gesagt sein, dass sich für den Roman kein einheitlicher Umgang mit der Shoah festmachen

5. - Hayden White, „The modernist event“, in: Vivian Sobchack (Hrsg.), The persistence of history. Cinema, Television, and the modern event, London/ New York, Routledge, 1996, S. 32.

6. - Wolfgang Hilbig, Das Provisorium, Frankfurt a.M., S. Fischer, $2008^{3}$, S. 153. Im Folgenden im Text zitiert als DP mit Seitenangaben. 
lässt. Allerdings liegt gerade in dieser Heterogenität der zentrale Reiz einer Auseinandersetzung.

\section{Die Spuren der Moderne}

Die naheliegendste Möglichkeit, den Spuren der literarischen Moderne in Hilbigs Werk nachzuforschen, ist diejenige der Intertextualität. Im Kontext der Autothematizität des Romans und der Parallelen zwischen dem Schriftsteller C. und dem Autor Hilbig sind diese intertextuellen Verweise, Anspielungen und Zitate nicht nur ausschmückendes Element, sondern das Bekenntnis zu einer Tradition modernen Schreibens. Die Untersuchungen zur Intertextualität in Hilbigs Werk, vor allem im Hinblick auf die ältere Lyrik und Prosa, sind Legion. So ist es nicht verwunderlich, dass sich auch in diesem Roman intertextuelle Verweise in fast unüberschaubarer Masse finden: Da taucht wieder die für den Autor fast schon obligatorische Hommage an Arthur Rimbaud auf, nämlich in Form eines Porträts, das man ihm in DDR-Zeiten gestohlen hatte, weil es ,aus dem Westen“ war. Die Parole "Ich ist ein anderer" habe der Schriftsteller C. schon lange bewundert, bevor sie zum „Welt-Klischee“ verkam $(D P, 91)$. Hilbig geht bis zum Zitat, wenn er Ezra Pound gegen die Konsumliteratur in den Kampf schickt $(D P, 71)$. Das Bewusstsein von der Unwiederbringlichkeit der Avantgarden, und vor allem des Surrealismus, spricht aus C.s Wahnvorstellungen, die ihn während einer Lesereise in Paris ereilen, als er im Delirium eine „graugrünweiß[e]“ Trikolore „von surrealen und mutierten Kadavern" erblickt $(D P, 147)$. Und doch bleibt die Zeit festgefroren: Die Bäume am Arc de Triomphe scheinen seit dem Foto von Hitlers Einmarsch nicht weitergewachsen zu sein $(D P, 149)$. Weitere intertextuelle Auftritte erlebt die literarische Moderne im Kondensat der Walter-Benjamin-Bände (DP, 179), und der Boxer-Schriftsteller Arthur Cravan weist erstaunliche Parallelen zu C. auf (DP, 229). Die literarische Moderne materialisiert sich schließlich als ein literarischer Schatz, der ebenso wie die Utopie mit dem Ende der DDR für C. sein „symbolisches Kapital 7" verloren hat:

[U]nd hinter dieser staubigen Lampe [sah er] die Bücher, die er immer gehütet hatte, wie seinen Augapfel: Lyrikbände aus westdeutschen Verlagen, Apollinaire, Kavafis, T. S. Eliot, „Nadja“ von Breton; „Wie es ist“ und „Aus einem aufgegebenen Werk“ von Beckett (DP, 216).

7. - Wolfgang Emmerich, „Schicksale der Moderne in der DDR“, in: Sabina Becker und Helmut Kiesel (Hrsg.), Klassische Moderne. Begriff und Phänomen, Berlin/ New York, de Gruyter, 2007, S. 433. 
Durch den autothematischen Charakter des Werkes ergeben sich zudem direkte Stellungnahmen gegen literaturwissenschaftliche Klassifizierungen. In Bezug auf die Postmoderne heißt es: „Die Wahrheit war, er hätte es als Zumutung empfunden, wenn man ihn irgendwo eingeordnet hätte“ $(D P, 72)$. Die Verweise auf die literarische Moderne sind deshalb oft ambivalent. C. möchte sie einerseits gerne beerben, befindet sich andererseits aber in einer tiefen Verunsicherung über gedankliche Nähe und historische Ferne.

\section{Die Shoah und die Krise des schreibenden Individuums}

An dieser Stelle soll näher untersucht werden, welche Position die Shoah innerhalb des Romans und damit für das poetologische Selbstverständnis des Schriftstellers C. einnimmt. Die schriftstellerischen Legitimationsprobleme werden im Provisorium an die Rolle der Shoah gekoppelt.

In einem Gespräch bezeichnet der Autor Hilbig die Unruhe als seine existenzielle Lebenserfahrung, die ihn schließlich zum Schreiben gebracht hat ${ }^{8}$, und auch für den Schriftsteller C. bedingt die innere Unruhe ein Bedürfnis nach Freiheit, das sein Staat allerdings nicht duldet. Die Umstände drängen C. zu Lügen und Ausflüchten, zur „Drückebergerei“ $(D P, 282)$ und er sucht zugleich nach einer Legitimation für sein Verhalten: „Er jagte irgendeinem Popanz nach, irgendeinem Beweis, dem, daß er ein wirklicher Schriftsteller sei ... er jagte einer irrealen Vorstellung nach, einem Phantom, einer Idealfigur aus der Vergangenheit" (ebd. Hervorhebung im Original). Es ist nicht allzu abwegig, in diesem Ideal die Schriftsteller der literarischen Moderne zu erblicken, welche in C.s altem Bücherregal aufgereiht stehen. Jedoch kommt er nicht über die Dialektik des Anerkennungsprozesses hinaus: Seine Selbstanerkennung bleibt durch die fehlende Fremdanerkennung aus - die "Redaktionsstuben der DDR-Literatur" haben für sein Schriftstellertum kein Verständnis $(D P, 283)$. C. tritt die Flucht nach vorn an und transponiert seine Notlügen in eine prinzipielle Verlogenheit der äußeren Welt. Die zentrale Lüge ist hier das im Namen des Fortschritts verübte Verbrechen des 20. Jahrhunderts9.

„Dort unten“ in C.s Gedächtnis, in seinem Unterbewussten, zu dessen Erkenntnis er durch psychoanalytische Fachliteratur versucht vorzustoßen, ist „nur Verrohung, Lüge und Flucht, das einzig Sichere ist der Verlust" " $(D P, 270)$. Die Lösung liegt zu weit zurück in der Kindheit, um sich erinnern zu können. Alle Konflikte der Geschichte und Politik laufen zurück in den Abgrund von C.s eigenem Ich, aus dem das Schreiben resultiert. „Die Hölle dieser Kindheit war wortlos [...].

8. - Vgl. Sylvie Marie Bordaux, Literatur als Subversion..., a.a.O., S. 5.

9. - Vgl. DP, 255. 
Und ich begann diese schweigende Hölle mit Wörtern zu füllen ... mit einem winzigen Teelöffel, dem Löffelchen eines Kindergeschirrs“, und er setzt dieses mühselige Ausfüllen der Leere fort, noch als Arbeiter erscheint ihm seine Existenz als ,eine einzige Zeit der Lüge“ (DP, 270 f.). Die fehlende Berufungsinstanz zum Schriftsteller, der Mangel an einer „Art Sendungsbewusstsein“ $(D P, 276)$ bleibt das Problem. Im Vergleich mit den großen Dichtern der Vergangenheit merkt C. schokkiert, dass seine Biometrie alledem widerspricht, was für einen Dichter charakteristisch sei und sitzt damit einem verqueren Biologismus auf: Der Arbeiter C. ist zu vital und potent, um als Dichter durchzugehen. Die Absurdität dieser Beobachtung bleibt C. verborgen, er fordert für sich den doppelten Beweis des Schriftstellertums: den ersten für sich selbst und den zweiten für die Welt. ,[Z]usammengenommen wären die beiden Beweise eine Art Gottesbeweis gewesen. Einen solchen aber, soviel wußte man, gab es nicht" $(D P, 277)$.

Nun könnte gesagt werden, hierin liege der Ursprung der existenziellen Scham, die C. quält. Doch der Protagonist begründet sie in Bezug auf das für ihn einzig wichtige Wissen des 20. Jahrhunderts, das in Form der Bücherkisten in C.s Wohnung lauert:

Holocaust \& Gulag. Da standen sie, diese rauchenden Kisten, und sie hatten jeglichen Vorwurf an die Welt absorbiert. Angesichts der Kisten, zum Bersten gefüllt mit dem unausdenkbaren Entsetzen der Neuzeit, zerstreut zu stammelnder provisorischer Druckschrift, war jede Klage feige, kindisch, gegenstandslos geworden. Wer diese Kisten im Haus hatte, dessen Beschwerden klangen so lächerlich wie das Pfeifen einer Ratte. Man mußte sich schämen für jede Unzufriedenheit, sich hassen, wenn man noch Unglück empfinden konnte, und man mußte es verschweigen vor diesem Wahnsinn, der die Sprache würdelos gemacht hatte. Und dafür mußten die Mächtigen der Welt im Grunde dankbar sein, sie mußten ungeheure Dankbarkeit verspüren vor dem Phänomen Auschwitz... und sie hüteten es dementsprechend wie ihren Augapfel. Und er dachte dies nicht nur in Richtung Osten. Und wenn es das Phänomen nicht gegeben hätte, dann hätten sie es nachholen müssen ... aber das war etwas, was man nicht denken durfte, wenn man in der Gesellschaft nicht ganz durch den Rost fallen wollte. Damit werde ich allein bleiben, dachte er nach einer Weile, während der Zug mit ihm durch das Land raste (DP, $154 \mathrm{f}$., Hervorhebungen im Original).

Dies ist eine Schlüsselstelle für das Verständnis der Identitätsproblematik von C. Sie resultiert gerade aus dem „Phänomen Auschwitz“. Der Hass, den C. in seine Worte legt, begründet sich offenbar aus der Instrumentalisierung dieses „Phänomens ${ }^{10 “, ~ d a s ~ f u ̈ r ~}$

10. - Der vorliegende Artikel möchte nicht versuchen, die Frage zu beantworten, ob man Auschwitz als ein Phänomen bezeichnen kann oder nicht. Damit würde ein 
ihn zum Repressionswerkzeug der bestehenden politischen und gesellschaftlichen Systeme verkommen sei. Wer die konkreten Benutzer dieses Werkzeugs sind, das einer Kritik an der Gegenwart den Mund verbietet, bleibt vorerst im Unklaren. Die Repression stellt sich als eine internalisierte Stimme dar, deren wiederholtes ,man musste“ nur vage auf den äußerlichen Ursprung verweist. Zugleich bemerkt der Protagonist die Ungeheuerlichkeit dieses Verdachts einer Instrumentalisierung des „Phänomen Auschwitz“ und die Notwendigkeit seiner Geheimhaltung in der klassischen Manier des Verschwörungstheoretikers. Auch wenn Hilbig hier nicht explizit poetologische Fragen abhandelt, erscheint die Stelle doch als ein Hadern mit einem Adornoschen Verdikt über die Literatur nach Auschwitz, dem sich C. unterwirft, sei es aus Selbsthass oder aus Einsicht in die Rechtmäßigkeit dieses literarischen Maßstabes für eine Welt, in der alles andere Lüge ist. Indizien dafür, dass hier von der poetologischen Bedeutung von Auschwitz die Rede ist, liegen in der Parallele, dass auch C. etwas wie seinen Augapfel hütet, und zwar das Bücherregal der literarischen Moderne $(D P, 216)$. Ebenso zeigt sich eine Analogie in der Notwendigkeit, die sowohl in C.s Schriftstellertum als auch in dem „Phänomen Auschwitz“ liegt: „Ja, schon immer hatte er Schriftsteller werden wollen, sein Leben lang, und wenn es diesen Beruf nicht schon gegeben hätte, dann hätte er ihn erfinden wollen", so wie das „Phänomen Auschwitz" hätte nachgeholt werden müssen (DP, 181). Das "Phänomen Auschwitz" meint hier nicht mehr - das ist C.s Vorwurf Auschwitz selbst, sondern seine ideologische Konstruktion.

Die Gleichursprünglichkeit von C.s Legitimationsproblem und der poetologischen Normativität von Auschwitz zeigt sich in C.s steigendem Unvermögen einer literarischen und allgemein kommunikativen Vermittlung: Die eigene Sprache unterliegt denselben Hemmnissen, wie die Shoah-Sachliteratur in C.s Kisten:

[E]s schien eher so, als hätten seine Wörter einen unheimlich langen Weg zurückzulegen; und wenn er sie endlich ins Freie gestoßen hatte, waren sie derangiert, abgerissen, halb zerquetscht und von Heiserkeit entstellt. Seine Wörter waren auf ihrer langen Wanderung gealtert, gebrechlich geworden $(D P, 156)$.

Frappierend erscheinen hier die Ähnlichkeiten zu einer Deformierung der Sprache in den Konzentrationslagern selbst, wie sie zum Beispiel $\mathrm{H}$. G. Adler thematisiert. Dass alle Sprache nach Auschwitz „würdelos“ und falsch geworden ist, für die Bezeichnung der neuen Welt nicht mehr

Thema angerissen werden, das in seiner Brisanz einen eigenen Artikel verdiente. Wenn hier von einem „Phänomen Auschwitz“" gesprochen wird, so ist dies immer ein wörtliches Zitat des Romans, dessen Protagonist die Existenz der Shoah keinesfalls leugnet, auch wenn er an ihre Instrumentalisierung glaubt. 
taugt, findet sich als Denkfigur ebenfalls bei Imre Kertész ${ }^{11}$ in seinem Roman eines Schicksallosen, der sich wiederum intensiv mit Adorno auseinandergesetzt hat. Die Erneuerung der Sprache setzt aber bei C. nicht ein, es bleibt bei der Feststellung ihrer Überalterung.

Auf den Kampf einiger Literaten gegen die universale Geltung von Auschwitz, damit ihre ,eigenen Blödheiten wieder gelesen wurden ...“ (DP, 257), kann C. ebenfalls nur mit beißendem Spott reagieren. Er erkennt also die Geltung des „Phänomens Auschwitz“ an und leidet doch selbst unter den Folgen. Seine Klagen über die Benachteiligung des DDR-Bürgers erscheinen somit schon im Vorhinein relativiert und als scheinheilig denunziert: „C. war erstaunt, wie einfach diese Dinge hier im Westen waren; erneut dachte er: Sie haben hier alles, ich habe nichts! -“ $(D P, 198)$. Hatte der Erzähler zuvor noch allgemein von den „Mächtigen der Welt" gesprochen, kann er sich hier des Verdachts nicht erwehren, dass Auschwitz für die Eliten der DDR das letzte Mittel war, um Klagen an der Gegenwart im Keim zu ersticken (vgl. DP, 186). Zum Ersten verbindet der Erzähler hier also den Antifaschismus-Diskurs der DDR - in der Rede vom ,,antifaschistischen Staat“ (ebd.) - mit der Shoah und mit Auschwitz, die tatsächlich im offiziellen DDR-Diskurs schwach repräsentiert waren ${ }^{12}$. Zum Zweiten erscheint die Shoah gleichzeitig als Enttarnung der allgemeinen Lügen und als Propagandawerkzeug zur Inversion dieser allgemeinen Lügen in individuelle. Das erklärt die höchst ambivalente Haltung, die der Protagonist ihr gegenüber einnimmt.

Da der Autor Hilbig an dem Ideal einer Literatur festhält, die kritisch ist, nicht rein selbstreferenziell, sondern im Kontakt mit der Gesellschaft,

11. - So sagt Kertész in seiner Rede zur Verleihung des Literaturnobelpreises 2002: ,[Ich bitte] Sie zu bedenken, was aus der Sprache, aus den Worten im zwanzigsten Jahrhundert geworden ist. Wahrscheinlich ist es für den heutigen Schriftsteller eine der ersten und erschütterndsten Entdeckungen, daß die Sprache, so wie sie ist, quasi aus einer vor unserer Zeitrechnung liegenden Kulturepoche auf uns überkommen, einfach untauglich zur Darstellung der wirklichen Prozesse und einstmals einhelliger Vorstellungen ist." Imre Kertész, „Heureka!“, in: Ders., Die exilierte Sprache. Essays und Reden. Mit einem Nachwort von Péter Nádas, Frankfurt a.M., Suhrkamp, 2003, S. 244 f.

12. - Carola Hähnel-Mesnard und Katja Schubert plädieren für eine Nuancierung, wenn es um die faktische Präsenz von Shoah und Auschwitz im Antifaschismus-Diskurs der DDR geht. In Abgrenzung von Wolfgang Emmerich verweisen sie auf die (wenn auch periphere) Existenz von literarischen Texten, die sich den Themen der Shoah widmen, ohne im Sinne des Antifaschismus-Diskurses instrumentalisiert werden zu können. Vgl. Carola Hähnel-Mesnard, Katja Schubert, „Störfall Holocaust. Eine Leerstelle in der ostdeutschen Literatur nach 1989? Antworten an Wolfgang Emmerich“, in: Dies., Störfall? Auschwitz und die ostdeutsche Literatur nach 1989, Berlin, Frank \& Timme, 2016, S. 12. Dagegen richte sich der Begriff „Holocaust“, wenn er im Roman vorkomme, eher an westdeutsche Diskurse wie Bénédicte Terrisse betont. Vgl. Bénédicte Terrisse, „Auschwitz in Wolfgang Hilbigs Texten aus dem Westen. Eine literarische Provokationsgeschichte“, in: Ebd., S. 64. 
die sie anklagt, erscheint dieses Verbot der Klage für den Schriftsteller C. als existenzbedrohend. Literatur soll kein Problemlösungsinstrument sein, aber „sie wollte die Dinge zur Sprache bringen - provozierend oder protestierend -, sie wollte sich immerhin am Unglück dieser Welt beteiligen 13“. Die Klage war für den Protagonisten immer mehr zu der einzigen Art und Weise von Teilhabe an Gesellschaft geworden. Ihr Verbot zwingt den Einzelnen in die Isolation. Doch selbst in der Isolation wird er nicht mehr in Ruhe gelassen: Wie bereits angedeutet, gebärdet sich die Ideologie, die der Erzähler den „Mächtigen der Welt““ und dem „antifaschistischen Staat“ zuschreibt, für C. als eine innere Gegenstimme, die ihn der Lüge bezichtigt, sobald er an die Möglichkeit von individueller Entfaltung glaubt. Dies zeigt sich auch noch dann, als der ideologische Apparat der DDR bereits zusammengebrochen ist. Die Klagen bleiben, sie können sich aber nun nicht mehr gegen einen spezifischen Staatsapparat richten, sondern fallen zurück auf den Klagenden selbst, weil er unfähig ist, die innere Gegenstimme wieder loszuwerden ${ }^{14}$. Dieser Vorgang einer internalisierten Repression generiert schlussendlich im Protagonisten erneut rassistische Minderwertigkeitsgefühle. Der Missbrauch des „Phänomens Auschwitz“ ermöglicht also die Reproduktion seiner Entstehungsbedingungen:

[E]s schienen in ihm die Argusaugen ganzer Staatsreligionen über die Ehrlichkeit seiner Gefühle zu wachen. [...] offenbar stammte er von einer minderwertigen Sippe ab, in der jeder Versuch einer Gemeinsamkeit in Verwahrlosung verrottet war... [...] Jetzt waren der Alkohol und seine Lektüre psychotherapeutischer Literatur [...] eine Allianz eingegangen: er fühlte sich vollends als Untermensch und hatte auch, schwarz auf weiß, die Beweise dafür $(D P, 295)$.

Diese Ambivalenz, dass Auschwitz zugleich als ein Werkzeug der Unterdrückung und als die einzige Wahrheit, beziehungsweise ein Ankerpunkt für die Ausbildung von schriftstellerischer Identität, dargestellt wird, macht die Anwendung moderner Darstellungsweisen unerlässlich. C. findet Realität nur noch in der universalen Katastrophe, die, sobald feste Narrative und Strukturen vorliegen, wieder zum Unterdrückungsmittel wird. In der Konsequenz muss der Protagonist schwanken und das Provisorium aufrechterhalten ${ }^{15}$, kann sich weder

13. - Wolfgang Hilbig, Abriss der Kritik. Frankfurter Poetikvorlesungen, Frankfurt a.M., Fischer-Taschenbuch-Verlag, 1995, S. 94.

14. - Vgl. André Steiner, Das narrative Selbst - Studien zum Erzählwerk Wolfgang Hilbigs. Erzählungen 1979-1991 . Romane 1989-2000. Frankfurt a.M., Peter Lang, 2008, S. 255.

15. - Carl Pietzcker bezieht dieselbe Beobachtung noch enger auf das Verhältnis von Identität und Erinnerung. Carl Pietzcker, „Grenze, Erinnerung und Literatur. Wolfgang Hilbigs Roman Das Provisorium“, in: Bernd Neumann, Dietmar Albrecht 
der Kausalität noch der Chronologie anvertrauen, Utopie und Telos hat er als Lüge enttarnt.

\section{Shoah und Gulag, West- und Ostdeutschland}

Es ist auffällig, dass Wolfgang Hilbig in seinem Roman den Gulag gleichberechtigt neben die Shoah stellt. Das Symbol des Viehwaggons, das Lager und all die anderen metonymischen Anspielungen, die Hilbig reichlich in seinen Text einbaut, rekurrieren oft auf eine Art allgemeines Verbrechen des 20. Jahrhunderts. Dieses vage allgemeine Verbrechen ist in der Welt des Schriftstellers C. überall präsent:

Das ganze Jahrhundert sei ein einziger Zug von Lügen gewesen, hatte er zu Hedda gesagt, in Form einer Lüge und beladen mit Lügen sei dieser Zug vorwärtsgefahren, durchgefahren, vorübergefahren, mit einer Lokomotive als Führungssymbol... [...] Der Schienenstrang für diesen Zug sei die Fortschrittslüge gewesen. Und der Zug habe die Viehwaggons durch das Land geschleppt, Viehwaggons voller Menschen, kaum noch als Menschen erkennbar, Richtung Auschwitz, Workuta, Majdanek, Magadan, unter einem Himmel, der ein Gespinst aus Lüge war (DP, 255, Hervorhebung F.G.).

Hilbig ist fast penibel darauf bedacht, die Lager des Nationalsozialismus, Auschwitz und Majdanek, mit den Lagern der UdSSR, Workuta und Magadan, aufzuwiegen. Das metonymische Gestaltungsmittel des Zuges wird zur Allegorie einer gesellschaftlich-technischen Moderne, gegen die die literarische Moderne anschreibt. Dass die Menschheit in einem ,riesigen Müllhaufe[n] von Lügen steckengeblieben“ (ebd.). sei, dient dem Protagonisten im Anschluss einzig als Begründung der eigenen Unaufrichtigkeit gegenüber seiner Geliebten und nimmt somit fast schon satirische Züge an: Die Entindividualisierung der Lagersysteme dient C. hier bloß zur Rechtfertigung der eigenen Egomanie. Deutlich wird hieran wieder der Rückbezug des Lügenbegriffs auf das Individuum C. Die Lüge ist nicht bloß eine ideologische, sie haftet dem Einzelnen direkt an. Sie wird zur emotionalen Lüge: C. ist ein Mann, der von den Frauen nicht loskommt, sie aber auch nicht lieben kann ${ }^{16}$.

und Andrzej Talarczyk (Hrsg.), Literatur. Grenzen. Erinnerungsräume. Erkundungen des deutsch-polnisch-baltischen Ostseeraums als einer Literaturlandschaft, Würzburg, Königshausen \& Neumann, 2004, S. 374.

16. - In ihrem Nachwort zur Ausgabe von 2013 rückt Julia Frank den Begriff der Lüge somit auch einzig in den Kontext der Liebe. Julia Frank, „Nachwort“, in: Wolfgang Hilbig, Das Provisorium. Roman, Frankfurt a.M., S. Fischer, 2013, S. 315. 
Dass der Schriftsteller C. höchstens in der zeitlichen Folge zwischen Shoah und Gulag unterscheidet, wird deutlich, wenn er die Argumentation des Roman-Verdiktes nach Auschwitz resümiert:

Gott hatte sich mit Grausen abgewandt vom Schund der RomanLeben, ab und zu deutete er mit dem Finger auf das Leben derjenigen, die Auschwitz hinter sich gebracht hatten. Denkende Menschen warfen die Bücher in die Mülltonne, die Antiquariate kauften das klebrige Zeug nicht mehr an. Mit der Unschuld des Erzählens war es vollkommen vorbei, seit es die Berichte aus dem Gulag gab (mit denen die Buchverlage übrigens ein Riesengeschäft machten) $(D P, 257)$.

Den zweifachen Gottesbeweis für das Schriftstellertum wiederaufnehmend, offenbart sich die hier mit Gott assoziierte Kritik als eine Position, die sich C. gläubig aneignet. Es ist die Position einer schriftstellerischen Avantgarde, die sich über die Unzulänglichkeit des klassischen Romans bewusstgeworden ist. Es ist zugleich die Position jener frühen Zeugenberichte aus den Konzentrationslagern, die alle Fiktion als Lüge abtut, und hier muss sich $\mathrm{C}$. wieder bedrängt fühlen, denn sein Schreiben ist, wie oben schon deutlich geworden, ein fiktionales und sein Protest ist die Lüge.

Der Hieb gegen die Kommerzialisierbarkeit selbst der GulagSachliteratur leitet schon über zum Vergleich der Shoah mit den zwei deutschen Staaten und ihren weltanschaulichen Systemen. An einer recht markanten Stelle des Textes ist vom „Lager“ die Rede, doch sind es weder diejenigen des Nationalsozialismus noch die des Gulag, sondern Flüchtlingsauffanglager des Jahres 1945, in deren Provisorium auch Hedda Rast, die russischstämmige Geliebte des Protagonisten, für kurze Zeit interniert war $(D P, 200)^{17}$. Viele, vor allem Westdeutsche, hätten in ihrer Klassifizierungswut später nicht begreifen wollen, dass hier nicht bloß Nazi-Kollaborateure versammelt waren.

Die Deutschen meinten, die Geschichte, die sie durcheinandergewürfelt hatten, wieder in Ordnung zu bringen, indem sie nach der Parteizugehörigkeit des menschlichen Abfalls fragten, den sie durch ganz Europa gehetzt und geprügelt hatten. Die Herrenmenschen des deutschen Wirtschaftswunders kamen ohne ihre Untermenschen nicht aus $(D P, 202)$.

Dass C. die Kontinuitäten zur Shoah vor allem auf der Seite der westdeutschen Konsumkultur zu entdecken meint, lässt sich vielfach nachweisen, auch in der Vokabel von der „Effizienz“, die Hilbig vom

17. - Sie ist das Alter Ego von Hilbigs Lebensgefährtin Natascha Wodin, einer russisch-deutschen Autorin. 
Lagerapparat auf die angebliche Konsumblödigkeit der BRD überträgt, wovon die DDR noch einiges habe lernen können $(D P, 141)$.

Besonders eklatant wird der Auschwitz-Vergleich in einer längeren traumartigen Passage, die in der Haupteinkaufsstraße von Nürnberg spielt. Die Wahl des Traums als Darstellungsmittel lässt an eine surrealistische Reminiszenz denken. Möglicherweise deutet sie aber auch auf eine Distanzierung von der oberflächlichen Textbedeutung hin.

Im Halbschlaf sah er sich mitten auf der Breiten Gasse stehen, zwischen seinen beiden Bücherkisten [...]; rings um ihn wallte das von der Nachmittagssonne beschienene Leben, die unaufhörliche gottgefällige Feier von Shopping \& Fun. Schon lange stand er so, er sah ziemlich abgekämpft aus, unrasiert, ungewaschen und etwas schmierig... worauf wartete er: er wartete auf den Abtransport. - Aber niemand achtete auf ihn; links und rechts von ihm gerierte sich die Konsumrevolution, der absolute Zeitgeist stelzte durch den Sonnenbrei. - Der würde, so sagte sich C., drüben in der DDR in tausend Jahren noch nicht ankommen... die Breite Gasse in Nürnberg, das ist der Nabel der Welt (oder die Königsstraße in Stuttgart, in Frankfurt die Zeil, in West-Berlin die Tauentzienstraße, oder die Untergrundmärkte auf den Bahnhöfen). Denn die Welt ist dort, wo sich Käufer und Verkäufer mit leuchtenden Gesichtern am Eingang zum Elysium begegnen... Shopping macht frei, so steht es in attraktiven Lettern über all diesen Eingängen zu lesen... (DP, $262 \mathrm{f}$.).

Die grammatische Parallele von ,Shopping \& Fun“ zur Betitelung der Bücherkisten (vgl. DP, 154) lässt die Stoßrichtung bereits erahnen, doch sie wird endgültig sichtbar in der an Sarkasmus kaum mehr zu überbietenden Anspielung des Erzählers auf den Schriftzug über dem Lagertor von Auschwitz als Eintritt ins Elysium. Wenig später treibt Hilbig diese Allegorie noch weiter. In demselben Traum entspinnt sich ein Dialog von Markenartikeln über den günstigsten Preis:

... wo gibt es denn solche Schnäppchen zu machen? - Aber echt, es ist wahr, mischt sich Nike Nr. 174517 ein (das ist zufällig dieselbe Nummer, die auf dem linken Unterarm von Primo Levi eintätowiert war, denkt C.) $(D P, 264)$.

Auch hier bedient sich Hilbig nur aus dem Anspielungsfundus der nationalsozialistischen Lager. Jedoch sagt diese Szene mehr über die Verlorenheit und elementare Verstörung des Protagonisten in einer Welt aus, die ihm ähnlich fremd erscheint wie die Konzentrationslager, als über eine tatsächliche Wesensverwandtschaft von Shoah und Konsumkultur. Dies lässt sich aber für das gegen Ende des Romans geschilderte Einfallen der westdeutschen Kapitalisten nach der Wende nicht mehr behaupten. Die Reminiszenzen an 1933 erscheinen vielmehr 
historisch. Möglicherweise bleibt hier die verstärkte Fiktionalisierung in der Form des Traums - deshalb aus, weil die Shoah nur hintergründig mitgedacht wird und nicht im Zentrum der Analogie steht:

[D]as Wort Deutschland hing ihnen [den westdeutschen Investoren - F.G.] zur Schnauze raus, an ihr Herz aber hielten sie einen Koffer voller Aktien gepreßt; sie hatten schon 1933 erst abgewartet, was der kleine Dackel mit der schwarzen Rotzbremse für Pläne hatte, und das hatte sich ausgezahlt. Jetzt machte die Volkspolizei mit den jungen Nazis gemeinsame Sache, zumindest kuschte sie vor ihnen, und die Roten standen überall am Pranger; das verbesserte langsam das Klima für Investitionen $(D P, 316)$.

Trotz dieser eifrigen Vergleichsarbeit zwischen der Shoah und der Konsumkultur Westdeutschlands, bleibt für C. die Frage offen, ob nun der west- oder der ostdeutsche Staat sich verheerender auf seine Person und seinen Schreibdrang ausgewirkt hat. Denn in der DDR herrscht die Kontinuität zum Gulag vor. Die ,ölige stumpfe [gelbe] Farbe“ (DP, 228) dient als tertium comparationis zwischen dem Grenzübergang Friedrichstraße und dem zentralen Verwaltungsgebäude des Gulagsystems, der Lubjanka in Moskau.

Wenn in den Büchern, die er gelesen hatte (aus der Abteilung Holocaust \& Gulag), der Begriff Lubjanka vorkam, dann hatte er die Gänge dieses Gefängnisses immer in dieser Farbe vor sich gesehen. Die Lubjanka war es, die in seiner Vorstellung diesen kalten, klebrigen, zur Erblindung führenden Farb- und Chloroformgeruch ausströmte, und der Geruch hatte sich bis zur Westgrenze der sogenannten DDR ausgedehnt und würde das Land nie wieder verlassen... (ebd.).

Nach dem Grenzübertritt nach West-Berlin vertreibt C. sich durch Whiskytrinken mit den Obdachlosen in der S-Bahn den Chloroformgeruch. Es ist wiederum der Geruchsinn im Spiel, wenn sich C. am Romanende im Leipziger Hauptbahnhof an die Deportationen erinnert fühlt. Da ist von dem Geruch der Rampen die Rede, ,über die das Tier- und Menschenfleisch des Lebens verschoben und verladen worden ist" $(D P, 319)$. Es ist die Zeit nach der Wiedervereinigung. C. betrachtet im Dämmerlicht die Fensterfront des Hauptbahnhofs ,und das siegreiche Zeichen gab der aufgehenden Sonne ihren Namen... AEG“ (DP, 320). Mit diesem Schlusssatz macht Hilbig noch einmal mehrere mögliche Vergleiche auf, zu den Zwangsarbeiterprozessen gegen die „Allgemeine Elektricitäts-Gesellschaft" in den 1990er Jahren, damit zur Ausbeutung von Zwangsarbeitern in der DDR und im Nationalsozialismus. Die aufgehende Sonne ist ein gängiges Symbol des Totalitarismus. Es scheint folglich, als begegne C. der Wende mit 
einer in Sarkasmus gekleideten Angst vor dem Sieg des augenscheinlich totalitären Kapitalismus. Hans-Christian Stillmark vertritt die These, dass es dem Autor hier nicht so sehr um makrostrukturelle Fragen, als vielmehr wieder um die labile Position des Individuums geht.

Das Problem ist in erster Linie nicht der Staat oder die Gesellschaft [...], es ist das „Monster“, der „Dämon“ und die „Bestie“ in jedem Einzelnen, deren bedrohliche Latenz als Dasein ohne Herkunft und Leben ohne Geschichte zur Sprache gebracht und erlitten wird ${ }^{18}$.

In diesem Sinne relativiert Hilbig auch durch den Vergleich von Shoah und Gulag zu DDR und BRD nicht so sehr die historischen Ereignisse selbst, als vielmehr die subjektive Erkenntnisposition des Protagonisten. Gerade in der Historisierung seiner subjektiven Identitätskrise kann C. die scheinbar objektivere Faktizität der Geschichtsschreibung als Lüge abtun. Fakt ist für ihn bloß das Gefühl, dass die Wahrheit eine andere ist als das scheinbar Objektive.

\section{Was bleibt von der Einzigartigkeit?}

Zwar wiegen die Anspielungen auf die Shoah insgesamt schwerer als diejenigen auf den Gulag, doch lässt sich für den Roman nur nach der Einzigartigkeit von „Holocaust \& Gulag“ als einem gemeinsamen Gegenstand fragen. Einzigartig erscheint dieses Verbrechen des 20. Jahrhunderts in Bezug auf seine epistemologische Bedeutung für das schreibende Individuum: Shoah und Gulag seien die einzige Wahrheit in der den Schriftsteller C. umgebenden Lügenwelt. Daraus ergibt sich eine interessante Umstülpung der Erkenntnissituation: Die Wahrheit liegt für C. nicht in einer diesseitigen Realität, sondern in einem Jenseits, das gerade durch seinen „Wahnsinn“ $(D P, 154)$ und durch seine Enttarnung von Sinnlosigkeit diesen Rang erst einnehmen kann. Diese Einzigartigkeit ist immer diejenige eines abwesenden Sinnes: Der Leser bemüht sich vergeblich, herauszufinden, was hier singulär ist, was hier wahr ist, und an der restlichen Welt Lüge. Er kann dies nach formallogischen Gesichtspunkten auch gar nicht, denn der Satz „Alles ist Lüge“ muss sich selbst widersprechen. Nur seine offensichtliche Abwesenheit kann ihn ausdrücken, ohne ihn zugleich zu widerrufen. Im Roman äußert sich dies auch insofern, als C. die Bücher aus der ,Abteilung Holocaust \& Gulag" nicht lesen kann, ihre Präsenz quält ihn. Möglicherweise sind die Shoah und der Gulag erst dadurch zur einzigen Wahrheit geworden, dass sie in der Black Box verschwunden sind, in einer Kiste, deren Inhalt

18. - Hans-Christian Stillmark, „In der Begierde verloren. Zu Wolfgang Hilbigs Roman Das Provisorium“", in: Eva Lezzi und Monika Ehlers (Hrsg.), Fremdes Begehren. Transkulturelle Beziehungen in Literatur, Kunst und Medien, Köln/Wien/Weimar, Böhlau, 2003 (Literatur - Kultur - Geschlecht, Bd. 22), S. 104. 
nicht mehr zugänglich ist. In einem derart radikalen Verständnis von Wahrheit und Lüge bleibt dann auch kein Raum mehr für eine Rettung des „guten“ sozialistischen Utopie-Kerns, wie es zum Beispiel Christa Wolf und Heiner Müller noch versuchen.

Hilbig hat gewissermaßen die Wiedervereinigung von Ost und West auch auf der Ebene der Gründungsherausforderungen Shoah und Gulag vollzogen. Die Pauschalität, mit der der Autor hier vorgeht, verrät noch einmal, dass sein Interesse kein historisches, sondern ein poetologisches ist. Das große Verbrechen des 20. Jahrhunderts ist im Roman zum Mythos geworden. Sein ideologisierender Missbrauch eröffnet die Möglichkeit der repressiven Aneignung durch die „Mächtigen“ beider Seiten. Der Autor macht aber an anderer Stelle deutlich, dass derselbe Mythos auch die Bedingung eines funktionslosen Schreibens sein kann, das sich von einer Instrumentalisierbarkeit freimacht. Er ist dann nicht mehr Medium, sondern stetige Selbstschöpfung und -erhaltung ${ }^{19}$.

Es bleibt fraglich, ob sich für Das Provisorium so etwas wie ein funktionsloses Schreiben überhaupt diagnostizieren lässt. Der Autor übt fundamentale Kritik an normativen Ausdeutungen der Shoah ${ }^{20}$, gerade indem er die Unmöglichkeit von Klage und Kritik thematisiert. Er kritisiert die Auswirkung ihres ideologischen Missbrauchs auf das schreibende Individuum, ohne dass er sich von den Schreibweisen der literarischen Moderne und einer spezifisch modernen Topik der Identitätskrise verabschieden könnte. Zudem kann gerade die Autothematizität des Romans - Schreiben über das Schreiben, über die Furcht vor dem Ende des Schreibflusses in einer neuen deutsch-deutschen Umwelt - nicht als funktionslos oder gar hermetisch beschrieben werden. Hilbig sieht sich in den 1990er Jahren oft mit der Frage nach dem Einfluss der Wende und Westdeutschlands auf sein Schreiben konfrontiert und versucht augenscheinlich mit dem Roman, hierauf eine Antwort zu geben ${ }^{21}$.

In Wolfgang Hilbigs Provisorium findet sich kein widerspruchsfreier Umgang mit der Shoah. Durch die Parallelisierung mit dem Gulag und die relativierende Bezugnahme auf die Konsumkultur wird

19. - „Sein [des Mythos“ - F.G.] Thema ist immer wieder die Schöpfung, und dieses Thema ist gleichzeitig seine Wirkung, der Mythos verkörpert sein eigenes Thema, er ist das Phänomen, das nicht Medium ist [...]. Das Schreibenmüssen eines Schriftstellers erklärt sich womöglich daraus, daß seine Texte nicht mehr Medium für irgendwelche Weisheiten sind, sondern daß ihr Leben begonnen hat, weil ihr Gedächtnis sich zu erhalten im Begriff ist.“ Wolfgang Hilbig, „Der Mythos ist irdisch. Für Franz Fühmann zum 60. Geburtstag“, in: Ders., zwischen den paradiesen. Prosa. Lyrik, Leipzig, Reclam, 1992, S. 209.

20. - Dies gilt auch für normative Ansätze innerhalb der Rezeption von Adornos Diktum.

21. - Vgl. Wolfgang Hilbig, ,Zeit ohne Wirklichkeit. Ein Gespräch mit Harro Zimmermann“, in: Heinz Ludwig Arnold, Text + Kritik. Zeitschrift für Literatur, H. 123 Wolfgang Hilbig, München, edition text + kritik, 1994, S. 11. 
auf der inhaltlichen Ebene der Fokus weg von Aussagen über tatsächliche geschichtliche Kontinuitäten und hin auf den Erkenntnisstatus des Individuums gerichtet, das bei all seinen Klagen über die ideologische Verstellung der Realität selbst nicht mehr in die Black Box blicken kann. Die innere Gegenstimme einer gestorbenen staatlichen Ideologie verhindert über die Wiedervereinigung der beiden deutschen Staaten hinaus ein $\mathrm{Zu}$-sich-Kommen des Protagonisten. Nicht nur, dass er in seinen Klagen der Omnipräsenz von Lüge nicht entfliehen kann, er kann sich tatsächlich auch von der normativen Instrumentalisierung des „Phänomens Auschwitz“" nicht freimachen, denn auch er nutzt es als Werkzeug der Denunziation. Trotz allem bleiben die verschlossenen Kisten, bleiben Shoah und Gulag, gerade durch ihre Unerreichbarkeit die einzige Konstante im Provisorium. In ihrer Losgelöstheit von der restlichen Welt bei gleichzeitiger wirksamer Präsenz in ihr geraten sie also zum Orientierungspunkt für Hilbigs avantgardistisches Verständnis einer selbstreferentiellen und gleichzeitig kritischen Literatur: ,[Literatur darf sich] nicht irgendwelche Aufgaben stellen lassen, weder von der Politik, noch von der Gesellschaft. [...] die Literatur kann es sein, die der Gesellschaft ihre noch ungelösten Aufgaben stellt ${ }^{22 “ "}$ 